\title{
Dietary supplement education in a senior population
}

\author{
Kimberly G. Elder ${ }^{1}$, Sarah A. Nisly ${ }^{2,3^{*}}$ \\ ${ }^{1}$ Department of Pharmacy, Community Hospital East, Indianapolis, USA; \\ ${ }^{2}$ Department of Pharmacy, Indiana University Health, Indianapolis, USA; \\ ${ }^{3}$ Department of Pharmacy Practice, Butler University College of Pharmacy and Health Sciences, Indianapolis, USA. \\ Email: snisly@butler.edu
}

Received 26 August 2011; revised 9 October 2011; revised 2 November 2011.

\begin{abstract}
Background: Dietary supplements are widely used among United States senior citizens for various indications. Potential dangers with supplement use inclu-

clusions: Dietary supplements were commonly used by the study population for various indications. Education by pharmacists is an effective method to increase knowledge and awareness about dietary supplements among this population.
\end{abstract} de the lack of regulation by the Food and Drug Administration (FDA) and the possibility for drug-supplement interactions. Senior focused education may increase the safe use of dietary supplements by older adults. Objective: The primary objective of this study was to determine the effectiveness of a pharmacist driven educational seminar in a local senior population. Methods: Participants aged 55 years and older in one of three senior programs were eligible for inclusion. Initially, a needs-assessment interview was conducted at a health fair. At that time, interviews focusing on dietary supplement use were delivered to study participants. Following the health fair, interview responses were analyzed to determine the most commonly used dietary supplements. During the second phase of the project, an educational seminar focusing on general dietary supplement information and the most commonly used supplements in the phase one population was created and delivered. Pre- and postsurveys were administered at the seminar to gauge baseline knowledge and impact of pharmacist education. Additionally, results were analyzed for any change in attitudes about dietary supplements. Results: Forty-nine participants were interviewed about their use of dietary supplements at the initial health fair. Among these participants, $81.6 \%$ were taking at least one supplement. The most commonly used supplements were calcium $(n=23)$, multivitamins $(n=22)$, fish oil $(n=13)$, and vitamin $B(n=12)$. Approximately 180 participants attended the subsequent educational seminar. Knowledge statistically significantly improved from baseline for all six questions posed to study participants. Overall, the program was well received and attitudes about dietary supplements changed as a result of viewing the seminar. Con-
Keywords: Dietary Supplements; Herbals; Aged; Drug Interaction; Education

\section{INTRODUCTION}

\subsection{Background}

According to the Dietary Supplement Health and Education Act (DSHEA) of 1994, dietary supplements include vitamins, minerals, herbals, amino acids, enzymes, glandulars, and metabolites. DSHEA also defined the term "new dietary ingredient" as a vitamin, mineral, herb or other botanical, amino acid, enzyme or tissue from organs or glands, or metabolite that was not marketed prior to 1994 [1]. Major problems with dietary supplements include the lack of regulation by the Food and Drug Administration (FDA) and the potential for drug interacttions [2]. Products containing new dietary ingredients require safety data to be submitted to the FDA by their manufacturers before they can be marketed. At this time, the many products marketed prior to DSHEA do not have to prove safety to the FDA. By law, the FDA has the responsibility to prove that a dietary supplement is unsafe before it can be forced off the market [1]. Even though the regulations for dietary supplements are not stringent, many consumers have the false belief that regulations are in place. A 2002 Harris Interactive Poll found that almost $60 \%$ of those surveyed believed that a regulatory agency like the FDA approved these products prior to being sold to the public [3]. A belief that alternative therapies are safe because they are 'natural' has also been documented [4].

Several studies have demonstrated a $7 \%$ - 45\% incidence of potential supplement-drug interactions [4-9]. The most common interactions involved dietary supplements cal- 
cium, garlic, valerian, kava, ginkgo, and St. John's Wort and prescription products such as anticoagulants, antidiabetic agents, antidepressants, and sedatives [5,7,8]. A 2008 study found that $29 \%$ of United States adults aged 57 - 85 years used at least five prescription medications daily [6]. Additionally, Lee and colleagues found that only one-third of patients using dietary supplements had use documented in their medical record [4]. Considering the significant use of prescription products and negligible documentation of dietary supplement use, the potential for drug interactions is of real concern. Older adults may be especially at risk because they suffer from a variety of conditions that they may choose to self-treat with dietary supplements.

Despite the minimal documentation in patient medical records, the use of dietary supplements is common among consumers, with $40 \%$ prevalence rates reported in 2008 [5]. Among the aged population, usage rates as high as 50\% have been reported [6]. One study of assisted living facility residents demonstrated nutritional supplement and herbal product use of $32 \%$ and $14 \%$, respectively [7]. These products were being used for various conditions, including general wellbeing, pain, arthritis, gastrointestinal upset, head and chest colds, cardiovascular disease, and anxiety and depression [2,6,8]. Additionally, recommendations for vitamin and mineral supplementation by organizations such as the American Dietetic Association (ADA), National Osteoporosis Foundation (NOF), and American Heart Association (AHA) may also contribute to use of these products [10-12]. This widespread use has lead to a significant amount of money being spent on supplements, with almost $\$ 15$ billion spent on natural products compared to $\$ 48$ billion on prescription medications in 2009 [13].

Due to their extensive drug and dietary supplement knowledge, as well as universal accessibility, a pharmacist is a logical healthcare practitioner to help educate the senior population about this topic. Studies have shown that education by pharmacists can improve patients' knowledge about a disease state [14,15]. At the time of this study, the effect of pharmacist education about dietary supplements remains unknown.

\subsection{Study Population}

Catholic Charities is a non-profit organization located in downtown Indianapolis, Indiana providing a variety of service opportunities for adults aged fifty-five years and older. The three main programs are the Senior Companions, Foster Grandparents, and Retired \& Senior Volunteers with group demographics listed below. Senior Companions are low-income adults trained to assist other homebound, frail adults with activities such as house- keeping, transportation to medical appointments, and medication reminders. The 111 participants are 86\% African-
American and 95\% women, primarily between the ages of 66 and 84. The Foster Grandparent program connect adults with local children for tutoring and mentoring opportunities. The 64 volunteers are 92\% African-American and 89\% women. The Retired \& Senior Volunteer program provides participants with numerous volunteer opportunities. The 192 volunteers are 77\% Caucasian and $67 \%$ women. Each group meets on a regular basis, during which they receive encouragement, education, and socialization.

\subsection{Objective}

The primary objective of this study was to determine the effectiveness of educational seminars in an aged population. Secondary objectives included evaluating the indication and usage of dietary supplements among this population.

\section{METHODS}

This study was conducted after approval by the Butler University College of Health Sciences institutional review board and included adults aged 55 years and older. The study was designed to occur in two phases (Figure 1). The first phase focused on general dietary supplement education and initial data collection via a one-onone interview. The second phase involved a focused education session for a larger study population based on the data gathered during phase I. Data was gathered during phase II using a paper survey. It was then analyzed for effects of the education provided.

During phase I of the study, volunteers for the Senior Companion Program were invited to attend a health fair during one of their routine meetings. An educational poster on dietary supplements was created and presented, and samples of various calcium supplements were available to participants for tastings. The poster included information such as common dietary supplement examples,

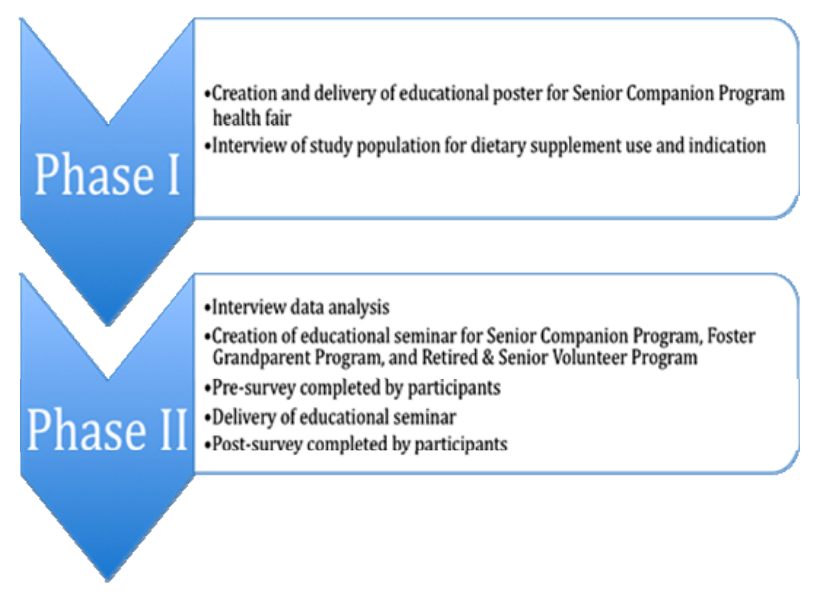

Figure 1. Study design. 
frequently asked questions, and recommendations on the use of dietary supplements with a specific focus on calcium supplements.

In addition to the poster, area pharmacists interviewed willing participants to collect basic demographic information, dietary supplement usage and indication, and additional perception information. The perception questions inquired about the perceived effectiveness of these products, physicians' awareness of product use, and number of prescription drugs used by participants. The interview format was used to avoid potential limited literacy. A sample of the data collection form is included in Appendix A.

Following the health fair, interview results were analyzed, and an educational seminar was created. The approximately forty-five minute seminar was presented via Microsoft ${ }^{\circledR}$ PowerPoint at an event for the members of the Senior Companion, Foster Grandparent, and Retired \& Senior Volunteers programs. The interactive seminar provided a general overview of dietary supplements, including information about their safety, the potential for drug interactions, and resources to learn more. It also provided information on the most reported supplements from the initial event. Information provided included indication, appropriate dosing, safety, and potential drug interactions.

Prior to the seminar, participants were given a presurvey that asked questions about demographics, content knowledge, and attitudes regarding dietary supplements. Participants completed the survey without assistance from members of the study team, and it was collected prior to delivery of the seminar. Following the seminar, participants completed a post-survey asking the same content knowledge questions, as well as attitude and evaluation questions. Pre- and post- patient surveys can be viewed in Appendix B.

\section{Statistical Analysis}

Interview and survey demographic, perception, and evaluation questions were analyzed using descriptive statistics in Microsoft ${ }^{\circledR}$ Excel 2003. Chi-squared tests were utilized to compare pre- and post-survey data.

\section{RESULTS}

\subsection{Phase I}

Forty-nine participants were interviewed about current use of dietary supplements. Demographics of the study population can be found in Table 1. Nearly $82 \%$ of participants reported taking at least once dietary supplement. The three most common supplements used by the study participants were calcium ( $\mathrm{n}=23,46.9 \%)$, multivitamin ( $\mathrm{n}=22,44.9 \%)$, and fish oil ( $\mathrm{n}=16,32.7 \%)$. Interview participants reported taking a mean of $5.3 \pm 4.3$ prescrip- tions on a daily basis. When examined by indication, $71 \%$ of participants utilized dietary supplements for general health. Sixteen percent of the study population utilized dietary supplements for cholesterol or triglycerides. Of the participants, $44.9 \%$ had noticed an improvement in their condition since the initiation of dietary supplements. Approximately two thirds of the study population reported informing their physician about dietary supplement use.

\subsection{Phase II}

Approximately 180 seniors attended the educational seminar, which included general information on supplements, as well as a focus on calcium, multivitamins, and fish oil. Participants completed varying parts of the preand post-surveys, with anywhere from 95 to 134 participant responses to the various questions. Demographics from this population can be found in Table 1. Statistically significant improvement was achieved for all knowledge questions (Table 2). Most participants either strongly agreed $(n=26,20 \%)$ or agreed $(n=64,49.2 \%)$ that the seminar changed their view of dietary supplements significantly. Likewise, participants either strongly agreed $(\mathrm{n}=17,13.7 \%)$ or agreed $(\mathrm{n}=58,46.8 \%)$ that they planned to change their use of dietary supplements following participation in the seminar. Ninety-four percent of participants either strongly agreed or agreed that the program met their educational needs, while $99.2 \%$ found the speaker to be effective.

Table 1. Baseline characteristics of participants in Phase I and II study populations.

\begin{tabular}{ccc}
\hline Demographic & Phase I & Phase II \\
\hline Mean Age, years \pm SD & $72.6 \pm 7.8$ & $72.3 \pm 7.9$ \\
Female, n (\%) & $48(98)$ & $126(94)$ \\
Ethnicity, n (\%) & & \\
Caucasian & $3(6)$ & $19(14.3)$ \\
African American & $44(90)$ & $49(36.8)$ \\
Hispanic & $0(0)$ & $64(48.1)$ \\
Asian & $0(0)$ & $0(0)$ \\
Other & $2(4)$ & $1(0.8)$ \\
Education, n (\%) & & \\
No High School Diploma & $16(33)$ & $27(20.3)$ \\
High School Diploma/GED & $16(33)$ & $45(33.8)$ \\
Some College & $14(29)$ & $43(32.3)$ \\
College Graduate & $3(6)$ & $12(9)$ \\
Graduate Degree & $0(0)$ & $6(4.5)$ \\
\hline
\end{tabular}


Table 2. Correct responses to knowledge questions

\begin{tabular}{cccc}
\hline Question & Pre-survey & Post-survey & p value \\
\hline & n (\%) & $\mathbf{n}(\%)$ & \\
1 & $58(51.8)$ & $83(66.4)$ & 0.022 \\
2 & $65(61.3)$ & $97(75.2)$ & 0.022 \\
3 & $27(23.3)$ & $85(65.9)$ & $<0.001$ \\
4 & $35(31.5)$ & $95(74.2)$ & $<0.001$ \\
5 & $64(60.4)$ & $98(76.0)$ & 0.01 \\
6 & $54(56.8)$ & $89(73.0)$ & 0.013 \\
\hline
\end{tabular}

\section{DISCUSSION}

This study examined the effectiveness of an educational seminar on dietary supplements in a population of senior adults. Results of the study support that the seminar did increase the knowledge of dietary supplements among this population. Participants answered knowledge questions about dietary supplements more accurately following the interactive seminar. A 2007 study also found that education by pharmacists on gastroesophageal reflux disease improved knowledge, although this study tested patients again at 8 - 12 weeks post education session [15].

Participants in phase II of the study overwhelmingly felt that participation in the seminar had changed their opinions on dietary supplements. They also stated that they planned to change their use of dietary supplements following the viewing of the seminar. While other studies have demonstrated the positive effect of pharmacist education, this study shows a focused impact of pharmacist education on dietary supplement knowledge. This education resulted in definite patient empowerment and ownership of medication management.

Dietary supplements were commonly used among the study population, with the most popular supplements being calcium, multivitamins, and fish oil. This may be due to various recommendations that national organizations have made regarding use of these products. For instance, the NOF recommends that all adults aged 50 years and older consume $1200 \mathrm{mg}$ of calcium each day [11]. The ADA recommends that vitamins and minerals are taken in via the diet but that supplementation is acceptable if diet sources are inadequate [10]. Finally, the AHA suggests consumption of two servings of fatty fish weekly to increase the intake of omega-3 fatty acids [12]. Awareness of these recommendations by physicians and the patient population alike may increase the utilization of these products by older adults.

This study is not without potential limitations. First, assessing study participants' improvement in knowledge immediately following the seminar may falsely inflate its perceived effectiveness. Time constraints and limited budget prohibited retesting participants at a later date. Also, variability among pharmacist delivery of interviews during phase I may have affected the information gathered from participants. To limit discrepancies, interviewers were debriefed on how to deliver surveys. However, the potential for variation remains. A final limitation was the potential for illiteracy in the second phase of our study. Participants in phase II were responsible for completing their own survey. Limited literacy may have contributed to incomplete surveys and inaccurate baseline demographics.

\section{CONCLUSIONS}

This study confirmed the prevalence of dietary supplement use among the older persons. It also shed light on the potential for pharmacist education sessions to teach this patient population about these products. It provoked a question as to whether survey methods are effective measures of knowledge in such a patient population due to potential problems with literacy. Future studies are needed to evaluate areas such as the ability of seminars to promote knowledge retention among participants, as well as the impact of individualized medication therapy management sessions for subjects who use both dietary supplements and prescription products.

\section{REFERENCES}

[1] Overview of Dietary Supplements (2010) Food and drug admi- nistration Website. http://www.fda.gov/Food/DietarySupplements/Consumer Information/ucm110417.htm

[2] Tachijian, A., Maria, V. and Jahangir, A. (2010) Use of herbal products and potential interactions in patients with cardiovascular diseases. Journal of the American College of Cardiology; 55, 515-525.

doi:10.1016/j.jacc.2009.07.074

[3] Harris poll shows widespread ignorance of supplement regulation.

http://www.supplementquality.com/news/Harris survey.h tml Published 23 December 2002. Accessed 5 September 2010.

[4] Lee, A.H., Ingraham, S.E., Kopp, M., et al. (2006) The incidence of potential interactions between dietary supplements and prescription medications in cancer patients at a Veterans Administration hospital. American Journal of Clinical, 29, 178-182. doi:10.1097/01.coc.0000209369.44100.25

[5] Sood, A., Sood, R., Brinker, F.J., et al. (2008) Potential for interactions between dietary supplements and prescription medications. American Journal of Medicine, 121, 207-211. doi:10.1016/j.amjmed.2007.11.014

[6] Qato, D.M., Alexander, G.C., Conti, R.M., et al. (2008) 
Use of prescription and over-the-counter medications and dietary supplements among older adults in the United States. Journal of the American Medical Association, 300, 2867-2878. doi:10.1001/jama.2008.892

[7] Lam, A. and Bradley, G. (2006) Use of self-prescribed nonprescription medications and dietary supplements among assisted living facility residents. Journal of the American Pharmacists Association (2003), 46, 574-581.

[8] Goldstein, L.H., Elias, M., Ron-Avraham, G.., et al. (2007) Consumption of herbal remedies and dietary supplements amongst patients hospitalized in medical wards. British Journal of Clinical Pharmacology, 64, 373-380. doi:10.1111/j.1365-2125.2007.02878.x

[9] Bush, T.M., Rayburn, K.S., Holloway, S.W., et al. (2007) Adverse interactions between herbal and dietary substances and prescription medications: A clinical survey. Alternative Therapies in Health and Medicine, 13, 3035.

[10] Marra, M.V. and Boyar, A.P. (2009) Position of the American Dietetic Association. Journal of the American Dietetic Association, 109, 2073-2085. doi:10.1016/j.jada.2009.10.020

[11] National Osteoporosis Foundation (2010) Clinician’s guide to prevention and treatment of osteoporosis. Available at http://www.nof.org/professionals/pdfs/NOF ClinicianGu ide2009 v7.pdf

[12] Penny, M.K., William, S.H. and Lawrence, J.A. (2002) Fish Consumption, Fish Oil, Omega-3 Fatty Acids, and Cardiovascular Disease. Circulation, 106, 2747-2757. doi:10.1161/01.CIR.0000038493.65177.94

[13] Nahin, R.L., Barnes, P.M., Stussman, B.J., et al. (2009) Costs of complementary and alternative medicine (CAM) and frequency of visits to CAM practitioners: United States, 2007. National health statistics reports; No. 18, National Center for Health Statistics, Hyattsville.

[14] Wang, K.Y., Chian, C.F., Lai, H.R., et al. (2010) Clinical pharmacist counseling improves outcomes for Taiwanese asthma patients. Pharmacy World and Science, Epub ahead of print.

[15] Finley, K., Giannamore, M., Bennett, M. and Hall, L. (2009) Assessing the impact of lifestyle modification education on knowledge and behavior changes in gastroesophageal reflux disease patients on proton pump inhibitors. Journal of the American Pharmacists Association (2003), 49, 544-548. 